\title{
Structure Analysis of a Highly Hydrophilic Recombinant Human-Source Gelatin
}

\author{
B. LIU, Y T. LEI and S L. YANG*
}

School of Environmental and Biological Engineering, Nanjing University of Science and Technology, Nanjing 210094, China

yshulin@mail.njust.edu.cn

Received 11 May 2012 / Accepted 27 June 2012

\begin{abstract}
Collagen and gelatin is widely used as bio-polymer materials and medical materials. Currently, the direction is more and more clear that collagen-like peptide produced by chemical synthesis, and recombinant collagen and gelatin produced in various expression system constructed by gene engineering technique substitute for these products prepared by traditional methods. Based on the characteristic of collagenous domain of human type III collagen, a recombinant human-source gelatin monomeric gene was designed and synthesized. After a series of gene cutting/ligation and transformation, etc. then expressed it in Pichia pastoris. The expression pruduct was purified from fermentation supernatant by gel filtration chromatography, then amino acid analyses, MALDI-TOF-MS analyses, FTIR spectroscopy, circular dichroism spectroscopy and scanning electron microscope were used to analyze the protein structure. It was confirmed that recombinant human-source gelatin is similar to animal-derived gelatin in protein structure.
\end{abstract}

Keywords: Recombinant human-source gelatin, Pichia pastoris, chemical synthesis gene, structure analysis

\section{Introduction}

Collagens and gelatins have been used widely in fields of food, cosmetic and medicine, etc ${ }^{1}$. Currently, most commercial collagens and gelatins are mainly prepared by acid hydrolysis or alkali-treating and enzyme-treating methods from such animal skins and bones as pigskin, fishskin and cattle cartilage ${ }^{2}$. However these products are liable to cause immunological reactions in human subjects and carry a risk of disease-causing contaminants and have some insurmountable disadvantages such as bad solubility and lots of hardships in separation and purification ${ }^{3}$. These problems greatly limit its application as biomedical material or drug delivery system, etc. With the development of chemical synthesis, molecular biology, transgenic technique and gene recombinant technique, various kinds of recombinant human or non-human collagens and gelatins have been produced.

The present study focuses on the expression of recombinant human gelatin in the methylotrophic yeast Pichia pastoris, which is deemed as a productive eukaryotic expression 
system ${ }^{4}$. In our laboratory, a recombinant human-source gelatin monomeric gene (Gel) encoding a highly hydrophilic $9 \mathrm{kDa}$ gelatin fragment was designed and synthesized according to human type III collagen. The gelatin fragment is made up of tandem repeats of "Gly-Xaa-Yaa” triplet sequences, and all hydrophobic amino acids ( proline excluded) are replaced by hydrophilic amino acids to improve the hydrophilic properties, and the codons encoding amino acids are replaced entirely according to Pichia pastoris bias usage to improve the expression level. After the construction of the recombinant vector pPIC9KG6 containing sixfold Gel (G6), the yeast GS115 (Pichia pastoris) was chosen to express the recombinant human-source gelatin encoded by G6. The expression of the recombinant Pichia pastoris reaches a very high level through high-density fermentation method. Then purified product was obtained by gel filtration chromatography. Here, we describe the structure analysis of the highly hydrophilic recombinant human-source gelatin by amino acid analyses, MALDI-TOF-MS analyses, FTIR spectroscopy, circular dichroism spectroscopy and scanning electron microscope.

\section{Experimental}

The expression product of the recombinant Pichia pastoris was obtained through high-density fermentation method. After purified from fermentation supernatant by gel filtration chromatography, the expression pruducts was used to subsequent analyse.

\section{Amino acid analyses}

The amino acid composition of recombinant human-source gelatin was determined by A200 amino Nova amino acid autoanalyser (membraPure, Germany) according to the standard of GB/T5009. 124-2003.

\section{MALDI-TOF-MS analyses}

Recombinant human-source gelatin was characterized by matrix assisted laser desorption/ionization time-of flight mass spectrometry (MALDI-TOF MS, Biflex III, Bruker, Germany). Sample was mixed with $5 \mu \mathrm{L}$ of trifluoroacetic acid (TFA, $0.1 \% \mathrm{v} / \mathrm{v}$ ) and $5 \mu \mathrm{L}$ of matrix solution (10 mg of sinapic acid in $1 \mathrm{~mL}$ of a 50:50 mixture of acetonitrile) and $0.1 \%$ TFA, (v/v). A volume of $1 \mu \mathrm{L}$ from this mixture was spotted on the target plate. Measurements were made in the positive, 1 inear mode and the accelerating voltage was $25 \mathrm{kV}$.

\section{FTIR spectroscopy}

Spectra were collected on an EQUINOX55 fourier transform infrared (FTIR) spectroscopy (Bruker, Germany) equipped with a DTGS detector. Solid sample was mixed with $\mathrm{KBr}$ in the mass ratio of 1 to 20 . All spectra were recorded at the resolution $4 \mathrm{~cm}^{-1}$ and 16 times scanning in the range of $4000-400 \mathrm{~cm}^{-1}$ at room temperature.

\section{Circular dichroism spectroscopy}

Protein was dissolved in ultrapure water at $0.1 \mathrm{mg} / \mathrm{mL}$. Measurements were performed on a Jasco J-810 spectropolarimeter. The pathlength was $0.1 \mathrm{~cm}$ and spectra were recorded from 190 to $260 \mathrm{~nm}$ at room temperature, using a scanning speed of $100 \mathrm{~nm} / \mathrm{min}$ at a resolution of $0.1 \mathrm{~nm}$.

\section{Scanning electron microscope}

The microstructures of lyophilized recombinant human-source gelatin was visualized by a JEOLJSM-6380LV scanning electron microscope (SEM) (JEOL Ltd, Japan) at accelerating voltage of $30 \mathrm{kV}$. 


\section{Results and Discussion}

Amino acid analyses

Amino acid analyses are shown in Table 1. Limited by the acid hydrolysis technique (During acid hydrolysis, Asn and Gln were transformed into Asp and Glu) and the resolution power of A200 amino acid autoanalyser, the results of amino acid analyses only present the numbers of six most amino acid composition, but it is enough to indicated that the amino acid composition of recombinant human-source gelatin is the same as the theoretical composition.

Table 1. Composition and molecular weight of recombinant human-source gelatin and human collagen III $(\alpha 1)$

\begin{tabular}{|c|c|c|c|c|c|c|}
\hline \multirow[t]{2}{*}{ Amino acid } & \multicolumn{2}{|c|}{$\begin{array}{c}\text { Recombinant } \\
\text { human-source gelatin } \\
\text { (detection value) }\end{array}$} & \multicolumn{2}{|c|}{$\begin{array}{c}\text { Recombinant } \\
\text { human-source gelatin } \\
\text { (theoretical value) }\end{array}$} & \multicolumn{2}{|c|}{$\begin{array}{l}\text { Human collagen } \\
\text { III }(\alpha 1)^{\mathrm{a}}\end{array}$} \\
\hline & Number & $\begin{array}{l}\text { Molar } \\
\text { ratio }\end{array}$ & Number & $\begin{array}{c}\text { Molar } \\
\text { ratio }\end{array}$ & Number & $\begin{array}{l}\text { Molar } \\
\text { ratio }\end{array}$ \\
\hline Ala (A) & $\mathrm{ND}^{\mathrm{b}}$ & ND & 6 & 1.00 & 91 & 8.32 \\
\hline $\operatorname{Arg}(\mathrm{R})$ & ND & ND & 0 & 0 & 49 & 4.48 \\
\hline Asn (N) & ND & ND & 72 & 12.02 & 25 & 2.28 \\
\hline Asp (D) & $62^{c}$ & $10.38^{c}$ & 0 & 0 & 30 & 2.74 \\
\hline Cys (C) & ND & ND & 0 & 0 & 3 & 0.27 \\
\hline Gln (Q) & ND & ND & 96 & 16.03 & 28 & 2.56 \\
\hline Glu (E) & $128^{c}$ & $21.49^{c}$ & 24 & 4.01 & 49 & 4.47 \\
\hline Gly (G) & 236 & 39.41 & 201 & 33.56 & 378 & 34.55 \\
\hline His $(\mathrm{H})$ & ND & ND & 0 & 0 & 7 & 0.64 \\
\hline Ile (I) & ND & ND & 0 & 0 & 17 & 1.55 \\
\hline Leu (L) & ND & ND & 0 & 0 & 22 & 2.01 \\
\hline Lys (K) & 15 & 2.44 & 18 & 3.01 & 40 & 3.66 \\
\hline Met (M) & ND & ND & 0 & 0 & 9 & 0.82 \\
\hline Phe (F) & ND & ND & 0 & 0 & 8 & 0.73 \\
\hline Pro (P) & 123 & 20.44 & 134 & 22.37 & 255 & 23.31 \\
\hline Ser (S) & 35 & 5.84 & 48 & 8.01 & 48 & 4.39 \\
\hline Thr (T) & ND & ND & 0 & 0 & 16 & 1.46 \\
\hline $\operatorname{Trp}(\mathrm{W})$ & ND & ND & 0 & 0 & 0 & 0 \\
\hline Tyr (Y) & ND & ND & 0 & 0 & 5 & 0.46 \\
\hline Val (V) & ND & ND & 0 & 0 & 14 & 1.28 \\
\hline Total & $599^{d}$ & 100 & 599 & 100 & 1094 & 100 \\
\hline $\begin{array}{l}\text { Molecular } \\
\text { weight } \\
(\mathrm{kDa})\end{array}$ & \multicolumn{2}{|c|}{$55.0^{\mathrm{d}}$} & & & \multicolumn{2}{|c|}{97.8} \\
\hline
\end{tabular}

\section{MALDI-TOF-MS analyses}

To obtain the accurate molecular weight of recombinant human-source gelatin, MALDI-TOF-MS analysis was performed to the purified sample. Figure 1 shows the results. 


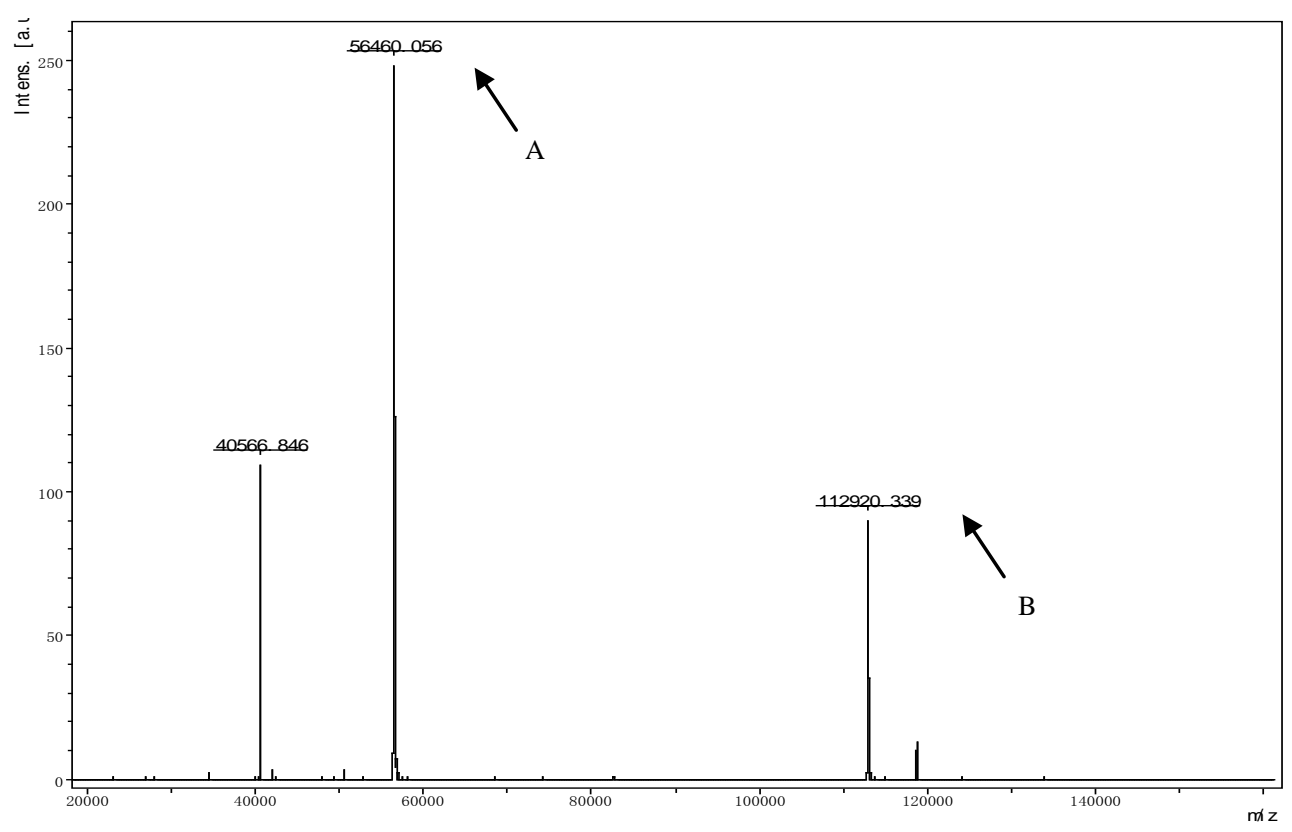

Figure 1. MALDI-TOF mass spectrum of recombinant human-source gelatin.

(The narrow A corresponds to the singly charged molecular ions of recombinant human-source gelatin monomer and the doubly charged molecular ions of recombinant human-source gelatin homodimer, The narrow $B$ corresponds to the singly charged molecular ions of recombinant human-source gelatin homodimer)

The highest peak indicated by narrow A is assigned to the singly charged molecular ions of recombinant human-source gelatin monomer and the doubly charged molecular ions of recombinant human-source gelatin homodimer according to the molecular weight of $56460.056 \mathrm{Da}$. Another peak indicated by narrow B is assigned to the singly charged molecular ions of recombinant human-source gelatin homodimer, its molecular weight (112920.339 Da) exactly twice that of peak A.

The observed accurate molecular weight of $56460.056 \mathrm{Da}$ is appreciably higher than its theoretical value of $55050.15 \mathrm{Da}$, which can be attributable to the post-translational modifications of both $\mathrm{O}$ - and $\mathrm{N}$-linked glycosylation ${ }^{5}$. This result also shows that the apparent high molecular weight observed in SDS-PAGE is indeed the result of aberrant migration behavior.

In addition, the molecular weight of recombinant human-source gelatin reachs the molecular weight range of native gelatin, which ensures the recombinant human-source gelatin is close to native gelatin in physical-chemistry properties.

\section{FTIR spectroscopy}

Infrared spectroscopy is one of the effective methods for the investigation of protein structure, which works on exploring the molecular mechanism of protein reactions and of protein folding, unfolding and misfolding. Collagen consists of three left-handed helical chains coiled around each other to form a right-handed supercoil. The collagen triple helix is stabilized by interchain hydrogen bonds. $\mathrm{N}_{\mathrm{G}}-\mathrm{H}_{\mathrm{G}}$ groups of the repeating -Gly-Xaa-Yaa- sequence of each 
chain are capable of forming hydrogen bonds with $\mathrm{C}_{\mathrm{X}}=\mathrm{O}_{\mathrm{X}}$ groups of adjacent subunit, and water-mediated hydrogen bonding links 4-hydroxyl groups belonging to 4-hydroxyproline with carbonyl groups in either glycine within the same chain or 4-hydroxyproline in the adjacent chain. Therefore the amide A, amide I, amide III of collagen own its notable spectroscopic characterization $^{6,7}$.

The FTIR spectra of sigma type III collagen (From calf skin) and recombinant human-source gelatin are shown in Figure 2, and the FTIR band assignments are shown in Table 2. The Table 2 displays that the wavenumbers of characteristic peaks of both are very adjacent from amide A, amide B in X-H stretching region (4000-2500 $\mathrm{cm}^{-1}$ ) to amide I, amide II in double-bond region (1900-1500 $\mathrm{cm}^{-1}$ ) and amide III in single-bond region (1500-600 $\mathrm{cm}^{-1}$ ). It shows that sigma type III collagen and recombinant human-source gelatin are similar in protein structure.

In X-H stretching region, both of sigma type III collagen and recombinant human-source gelatin display four peaks correspond to amide $\mathrm{A}$, amide $\mathrm{B}$, symmetric stretching vibration and antisymmetric stretching vibration of $\mathrm{CH}_{2}$ groups. The amide A of collagen arises mainly from the $\mathrm{N}-\mathrm{H}$ stretching vibration, its wavenumber depends on the strength of hydrogen bond, so it is very sensitive to the triple helix structure of collagen. The wavenumber of amide A of recombinant human-source gelatin and sigma type III collagen is $3308 \mathrm{~cm}^{-1}$ and $3301 \mathrm{~cm}^{-1}$ respectively, these less than those (3325-3330 $\mathrm{cm}^{-1}$ ) given by Doyle et $\mathrm{al}^{6}$. For collagen and a series of model polytripeptides, as pointed out by these authors, the amide A band of collage and other trple-helical polytripeptides always appears about $25 \mathrm{~cm}^{-1}$ higher than that observed for other secondary structures and can thus be used specifically to identify the collagen like structure. So, we can believe that both of recombinant human-source gelatin and sigma type III collagen are similar to denatured collagen in protein structure, all of them lack entire triple-helical conformation.

In the single-bond region, in addition to the absorptions due to stretches of single bonds, absorptions due to bends also occur in this region. There are usually so many bands here that it is difficult to assign each to a specific vibration. However, the large number of bands makes this region especially useful in comparing spectra, so it is also known as the fingerprint region. In this region, recombinant human-source gelatin and sigma type III collagen present obvious difference. We speculate that this difference root in their different amino acid residues. The sigma type III collagen derived from calf skin, but the amino acid sequences of recombinant human-source gelatin is based on human type III collagen $\alpha 1$ chain, and its gene had been modified.

Table 2. FTIR band assignments of sigma type III collagen and recombinant human-source gelatin

\begin{tabular}{ccc}
\hline \multicolumn{2}{c}{ Peak wavenumber $/ \mathrm{cm}^{-1}$} & \multirow{2}{*}{ Assignment } \\
\cline { 1 - 2 } Sigma type III collagen & Recombinant human-source gelatin & \\
\cline { 1 - 2 } 3301 & 3308 & Amide A \\
3078 & 3080 & Amide B \\
2941 & 2937 & $v_{\text {as }}\left(\mathrm{CH}_{2}\right)$ \\
2887 & 2887 & $v_{\mathrm{s}}\left(\mathrm{CH}_{2}\right)$ \\
1659 & 1661 & Amide I \\
1540 & 1541 & Amide II \\
1449 & 1450 & $\delta\left(\mathrm{CH}_{2}\right)$ \\
1404 & 1403 & $v\left(\mathrm{COO}^{-}\right)$ \\
1243 & 1242 & Amide III \\
\hline
\end{tabular}

$v$ : stretching vibration, $v_{s}$ : symmetric stretching vibration, $v_{a s}:$ antisymmetric stretching vibration, $\delta:$ in plane bending vibration 

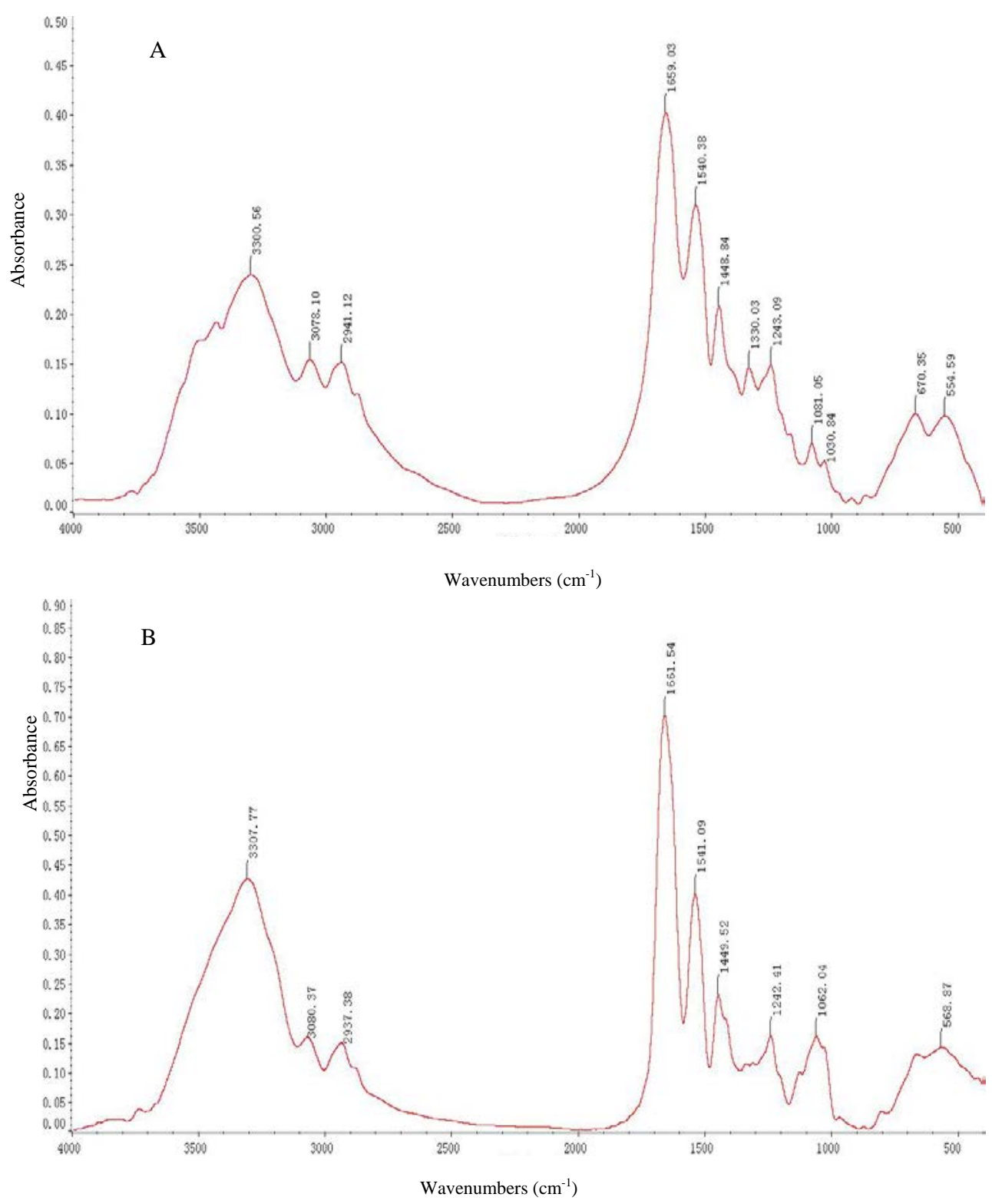

Figure 2. FTIR of recombinant human-source gelatin and sigma type III collagen (A. Sigma type III collagen, B. Recombinant human-source gelatin)

\section{Circular dichroism spectroscopy}

Circular dichroism spectroscopy was performed to validate whether the recombinant human-source gelatin forms triple-helix conformation. Figure 3 shows a clear absence of the positive peak at about $220 \mathrm{~nm}$ characteristic of the collagen triple helix ${ }^{8}$. This conclusion is identical to that of FTIR spectroscopy. 


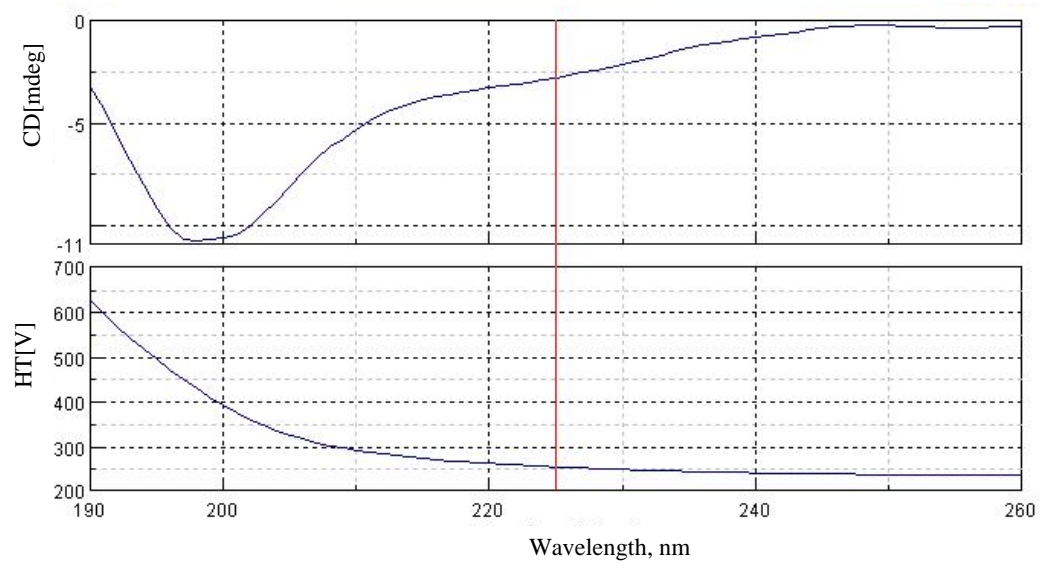

Figure 3. Circular dichroism spectroscopy of recombinant human-source gelatin

\section{Scanning electron microscope}

Four electron micrographs at 250, 500, 1500 and 5000× magnifications, respectively, displayed in Figure 4, show that no collagen fibrils are formed in lyophilized recombinant human-source gelatin, only a sponge-like structure are observed, which is consistent to the FTIR analysis result that the recombinant human-source gelatin lacks entire triple-helical conformation. But this sponge-like structure exactly indicates that the recombinant human-source gelatin is suitable for preparation of hemostatic sponge and artificial skin as scaffold material, etc.
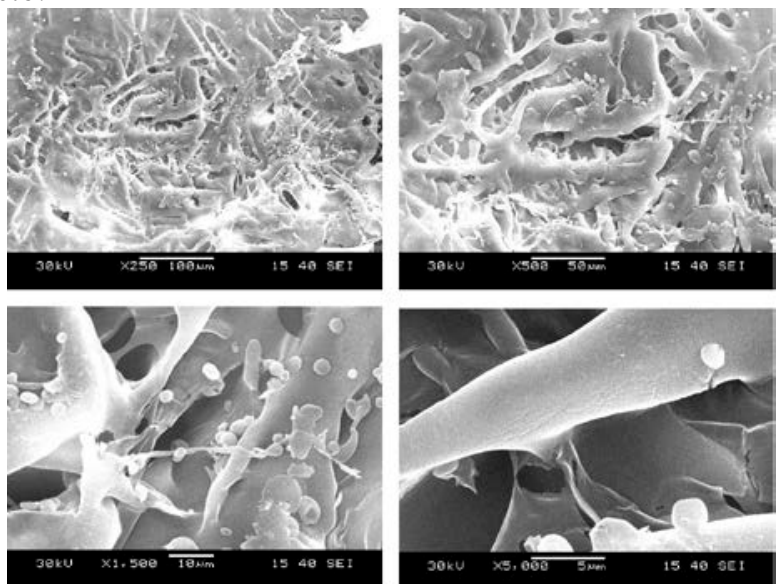

Figure 4. Electron micrographs of lyophilized recombinant human-source gelatin

\section{Conclusions}

Expression of new proteins by designing and chemical synthesizing sequences is superior in flexibility to full length cDNA cloning or partial sequences splicing method, therefore it could be considered to be a new way to prepare safe and new recombinant gelatins used in cosmetic, medicine and other fields. Amino acid analyses, MALDI-TOF-MS analyses, FTIR spectroscopy, circular dichroism spectroscopy and scanning electron microscope show that 
recombinant human-source gelatin mainly exists by homodimer and is prone to separate into monomer, and is similar to animal-derived gelatin in protein structure.

\section{Acknowledgement}

This study was supported by grants from National "863" Plan (No: 2008AA12A218) and Ph. D. Program Foundation of Ministry of Education of China (No: 20093219110013).

\section{References}

1. Young S, Wong M, Tabata Y and Mikos A G, J Controlled Release, 2005, 109, 256-274.

2. Gimenez B, Turnay J, Lizarbe M A, Monteroa P and Gómez-Guilléna M C, Food Hydrocolloid, 2005, 19, 941-950.

3. $\quad$ Bussemer T, Dashevsky A and Bodmeier R, J Controlled Release, 2003, 93, 331-339.

4. Cregg J M, Cereghino J L, Shi J and Higgins D R, Mol Biotechnol., 2000, 16, 23-52.

5. Cregg J M, Methods in Molecular Biology; Pichia Protocols, Second Edition, Volume 389, Humana Press, Totowa, N J, 2007.

6. Doyle B B, Bendit E G and Blout E R, Biopolymers, 1975, 14, 937.

7. Lazarev Y A, Grishkovsky B A and Khromova T B, Biopolymers, 1985, 24, 1449.

8. De Wolf F A and Keller R C A, Prog Colloid Polym Sci., 1996, 102, 9-14. 